\title{
GPS AND GLONASS COMBINED STATIC PRECISE POINT POSITIONING (PPP)
}

\author{
D. Pandey ${ }^{\text {a }}$, R. Dwivedi ${ }^{\text {a }}$, O. Dikshit ${ }^{\text {b }}$, A. K. Singh ${ }^{\text {a }}$ \\ ${ }^{a}$ Geographic Information System (GIS) Cell, MNNIT Allahabad, India -dwijpandey068@gmail.com, (ramjid, aksingh)@mnnit.ac.in \\ ${ }^{\mathrm{b}}$ Department of Civil Engineering, IIT Kanpur, India -onkar@iitk.ac.in
}

Commission I, WG I/5

\begin{abstract}
KEY WORDS: GPS, GLONASS, Precise Point Positioning (PPP)
\end{abstract}
\begin{abstract}
With the rapid development of multi-constellation Global Navigation Satellite Systems (GNSSs), satellite navigation is undergoing drastic changes. Presently, more than 70 satellites are already available and nearly 120 more satellites will be available in the coming years after the achievement of complete constellation for all four systems- GPS, GLONASS, Galileo and BeiDou. The significant improvement in terms of satellite visibility, spatial geometry, dilution of precision and accuracy demands the utilization of combining multi-GNSS for Precise Point Positioning (PPP), especially in constrained environments. Currently, PPP is performed based on the processing of only GPS observations. Static and kinematic PPP solutions based on the processing of only GPS observations is limited by the satellite visibility, which is often insufficient for the mountainous and open pit mines areas. One of the easiest options available to enhance the positioning reliability is to integrate GPS and GLONASS observations. This research investigates the efficacy of combining GPS and GLONASS observations for achieving static PPP solution and its sensitivity to different processing methodology. Two static PPP solutions, namely standalone GPS and combined GPS-GLONASS solutions are compared. The datasets are processed using the open source GNSS processing environment gLAB 2.2.7 as well as magicGNSS software package. The results reveal that the addition of GLONASS observations improves the static positioning accuracy in comparison with the standalone GPS point positioning. Further, results show that there is an improvement in the three dimensional positioning accuracy. It is also shown that the addition of GLONASS constellation improves the total number of visible satellites by more than $60 \%$ which leads to the improvement of satellite geometry represented by Position Dilution of Precision (PDOP) by more than $30 \%$.
\end{abstract}

\section{INTRODUCTION}

Global Navigation Satellite System (GNSS) is used to calculate the geographic location of a user anywhere in the world (Wellenhof, 2007; Overby, 2000). GNSS receivers using the GPS, GLONASS, Galileo or Beidou system are used in various applications such as navigation, surveying and mapping (Gao, 2007). Precise point positioning (PPP) is considered as a novel approach for providing centimetre to decimetre solution in quick time using current and coming GNSS constellations (Heroux, 2001; Gao, 2009). PPP requires less number of reference stations distributed around the globe rather than conventional differential approaches. PPP using a single receiver is a positioning method which calculates very precise positions up to few centimeter levels in a dynamic and global reference framework like International Terrestrial Reference System (ITRS) (Leick, 2004).

Currently, PPP systems are used to calculate point position using GPS observations only. Nevertheless, the accuracy and reliability of PPP results are dependent on the number of visible satellites (Hofmann-Wellenhof, 2007). Under the environments of mountains, urban canyons as well as open pit mines, for instance, the number of visible GPS satellites is often not sufficient for position assessment (Tsujii, 2000). Further, even in the open areas where sufficient GPS satellites are available, the accuracy and reliability of PPP solutions may still be insufficient due to very poor geometry of satellites in space (Cai, 2009). One of the probabilistic ways to increase the availability of satellites as well as the reliability of the positioning results is to make use of currently available satellite constellation of GNSS system by integrating the GPS and
GLONASS observations. The benefit from such integration is obvious particularly for applications in mountain, urban canyons and open pit mining environments (Cai, 2007).

Recently, Cai and Gao (2013) prepared an comprehensive analysis on modulating GPS and GLONASS observations for PPP in which numerical comparisons and analysis were explained using one day of static data from International GNSS Service (IGS) stations distributed around the globe and kinematic data gathered on a land vehicle. It is observed that the additional GLONASS observations could significantly reduce the solution convergence time. However, a better convergence time is still dependent on many factors such as the number and geometry of visible satellites, user environment and dynamics, observation quality and sampling rate (Bisnath, 2008). In addition, currently PPP is only capable of providing centimeterlevel accuracy in a static mode and decimeter-level accuracy in kinematic mode (Zhu, 2014). Further improvement of the positioning accuracy is still needed for some applications such as location based services (LBS). Since more visible satellites and observations are available, a mixed use of GPS and GLONASS observations in the PPP is expected to improve the positioning accuracy, reliability and convergence time behavior. Combining the observations from different GNSS could be an appropriate approach to fill this gap because the multiconstellation system guarantees improved satellite availability in comparison to a standalone GPS and hence enhancing accuracy, continuity and integrity of the positioning (Gioia, 2013).

In this paper, we investigate the efficacy of integrating GPS and GLONASS observations for improved accuracy and reliability of positioning results using PPP. The paper addresses the issues 
related to PPP and conducts a data analysis to assess its performance. Firstly, PPP model of combined GPS and GLONASS is described. A brief summary of processing methodology of $g L A B$ and magicGNSS is presented followed by analysis of results achieved. Results are compared for GPS (G) only and combined GPS and GLONASS (GR) processing.

\section{COMBINED GPS AND GLONASS PPP MODEL}

If we assume that the relativistic errors, satellites and receiver phase centre biases, site displacement effects (solid earth tides and ocean loading) and antenna phase wind-up have been properly considered, the pseudorange and carrier phase observables on GPS $L 1$ and $L 2$, and GLONASS $G 1$ and $G 2$ can be expressed as (Leick, 2004):

$$
\begin{aligned}
& P_{L_{i}}^{G}=\rho^{G}+c\left(d t^{G}-d T^{G}\right)+d_{\text {ION }}^{G}+d_{\text {TROP }}^{G}+\varepsilon_{P_{L_{i}}^{G}} \\
& \varphi_{L_{i}}^{G}=\rho^{G}+c\left(d t^{G}-d T^{G}\right)-d_{\text {ION }}^{G}+d_{\text {TROP }}^{G}+\lambda_{L_{i}}^{G} N_{L_{i}}^{G}+\varepsilon_{P_{L_{i}}^{G}} \\
& P_{G_{i}}^{R}=\rho^{R}+c\left(d t^{R}-d T^{R}\right)+d_{\text {ION }}^{R}+d_{\text {TROP }}^{R}+\varepsilon_{P_{G_{i}}^{R}} \\
& \varphi_{G_{i}}^{R}=\rho^{R}+c\left(d t^{R}-d T^{R}\right)-d_{\text {ION }}^{R}+d_{\text {TROP }}^{R}+\lambda_{L_{i}}^{R} N_{L_{i}}^{R}+\varepsilon_{P_{G_{i}}^{R}}
\end{aligned}
$$

Where the superscripts $G$ and $R$ denote a GPS and GLONASS satellite respectively; Li denotes GPS $L 1$ and $L 2$ frequencies; $G i$ denotes GLONASS $G 1$ and $G 2$ frequencies; $P$ is the measured pseudorange $(\mathrm{m}) ; \varphi$ is the measured carrier phase (cycle); $\rho$ is the true geometric range $(\mathrm{m}) ; c$ is the speed of light $\left(\mathrm{ms}^{-1}\right) ; d t$ is the receiver clock error $(\mathrm{s}) ; d T$ is the satellite clock error (s); $d_{I O N}$ is the ionospheric delay $(\mathrm{m}) ; d_{\text {TROP }}$ is the tropospheric delay $(\mathrm{m}) ; \lambda$ is the wavelength of the carrier phase measurements (m); $N$ is the non-integer phase ambiguity including the initial phase bias (cycle); and $\epsilon$ is the observation noise and residual multipath $(\mathrm{m})$.

For dual frequency PPP users, the ionospheric error can be eliminated to first order by forming the ionosphere-free combination (L3/G3) of the pseudorange and carrier phase observations. After applying the precise orbit and clock corrections, the ionosphere-free combinations can be expressed as follows (Leick, 2004):

$$
\begin{aligned}
& \varphi_{L 3}^{G}=\frac{1}{f_{L 1}^{2}-f_{L 2}^{2}}\left(f_{L 1}^{2} \varphi_{L 1}^{G}-f_{L 2}^{2} \varphi_{L 2}^{G}\right)=\rho^{G}+c\left(d t^{G}-d T^{G}\right)+d_{T R O P}^{G}+N_{L 3}^{G}+\varepsilon_{\varphi_{L 3}^{G}} \\
& P_{L 3}^{G}=\frac{1}{f_{L 1}^{2}-f_{L 2}^{2}}\left(f_{L 1}^{2} P_{L 1}^{G}-f_{L 2}^{2} P_{L 2}^{G}\right)=\rho^{G}+c\left(d t^{G}-d T^{G}\right)+d_{T R O P}^{G}+\varepsilon_{P_{L 3}^{G}} \\
& \varphi_{G 3}^{R}=\frac{1}{f_{G 1}^{2}-f_{G 2}^{2}}\left(f_{G 1}^{2} \varphi_{G 1}^{R}-f_{G 2}^{2} \varphi_{G 2}^{R}\right)=\rho^{R}+c\left(d t^{R}-d T^{R}\right)+d_{T R O P}^{R}+N_{G 3}^{R}+\varepsilon_{\varphi_{G 3}^{R}} \\
& P_{G 3}^{R}=\frac{1}{f_{G 1}^{2}-f_{G 2}^{2}}\left(f_{G 1}^{2} P_{G 1}^{R}-f_{G 2}^{2} P_{G 2}^{R}\right)=\rho^{R}+c\left(d t^{R}-d T^{R}\right)+d_{T R O P}^{R}+\varepsilon_{P_{G 3}^{R}}
\end{aligned}
$$

Where the $P_{L 3}$ and $P_{G 3}$ are the ionosphere-free combination of GPS and GLONASS pseudorange measurements respectively (m); $\varphi_{L 3}$ and $\varphi_{G 3}$ are the ionosphere-free combination of the GPS and GLONASS carrier phase measurements respectively $(\mathrm{m}) ; f_{i}$ is the GPS and GLONASS carrier frequency $(\mathrm{Hz})$. In equations (5-8), the unknown parameters are three position coordinates $(X, Y, Z)$ of the receiver, two constellation-specific receiver clock parameters, tropospheric delay (including tropospheric gradients) and an ambiguity for GPS and GLONASS satellites.

\section{PROCESSING OF GPS AND COMBINED GPS/GLONASS PPP}

To examine the feasibility of only GPS as well as combining GPS and GLONASS observations for PPP, we used the $g L A B$ 2.2.7 as well as magicGNSS software package. The GNSS-Lab Tool suite $(g L A B)$ is an interactive educational all-purpose package to process and examine GNSS data (http://gage.upc.edu/gLAB). The first display of this software package allows processing only GPS data, but it is prepared to consolidate future module updates, such as an elaboration to Galileo and GLONASS systems, EGNOS and differential processing (Sanz, 2011).

This software implements full processing capabilities for GPS data. However, the reading of RINEX-3.00 Galileo and GLONASS data functionality is also included, allowing performing some exercises on data examination with real or simulated Galileo and GLONASS measurements (Sanz, 2011). Figure 1 shows the working flowchart of $g L A B$ processing chain.

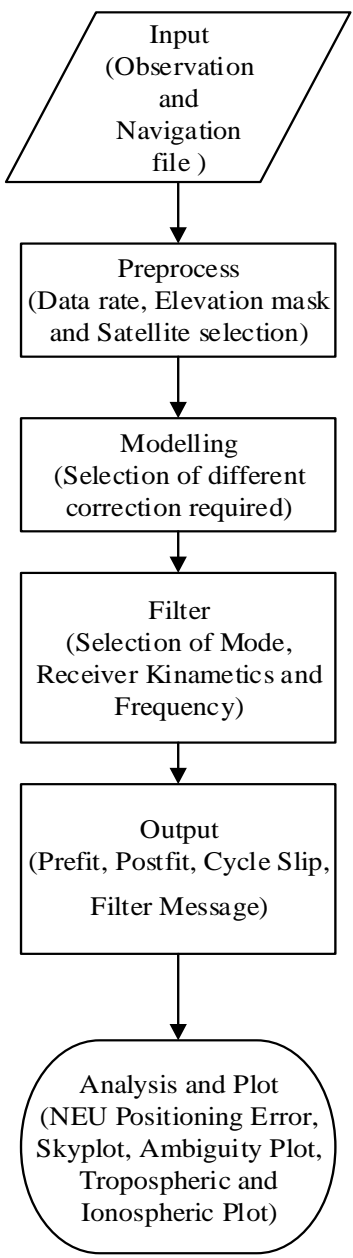

Figure 1: PPP processing using gLAB software package

On the other hand GMV Aerospace and Defense S.A. has announced its magicGNSS, a set of software resources that supports a wide variety of GNSS projects and goals, which includes service volume simulations, core operational functions (namely orbit, clock, ionosphere determination and prediction), receiver performance analysis, added value services including 
integrity, local augmentation advancements and all related execution as well as accuracy analysis (http://magicgnss.gmv.com/). A Receiver Performance Analysis (RXAN) software implements user receiver algorithms providing GNSS performance (GPS, GLONASS and Satellitebased Augmentation System (SBAS Systems) based on the user given true position coordinate. It can process data from several receiver models, RINEX navigation as well as observation files and SBAS) messages. It provides standard performance and integrity information. The tool set may be applied to the projects which relate GPS, Galileo, GLONASS as well as their augmentation systems. GMV also offers magicGNSS Beta, an open source online service for registered users. At present it provides a demo version of the Orbit Determination and Time Synchronization (ODTS) module supporting GPS and GIOVEA and B (Galileo) data (Sanmartín, 2012). Figure 2 explains the methodology of mixed (GR) PPP processing.

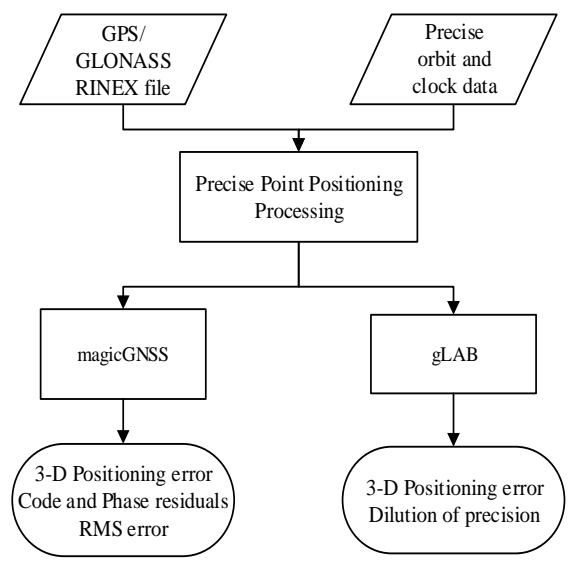

Figure 2: Methodology of combined (GR) PPP processing

\section{RESULTS AND DISCUSSION}

To analyze the results of the combined GPS and GLONASS PPP, a two-hour dataset collected at Geospatial Training and Application Center (GTAC) on November 11, 2015 is downloaded to estimate the station position (http://geodesy.noaa.gov/UFCORS/). The detailed information such as the satellite number, Position Dilution of Precision (PDOP), observation residuals and parameter estimation is estimated. The site coordinates from CODE Analysis Centers were used as a true coordinates to assess the accuracy of PPP (http://geodesy.noaa.gov/UFCORS/). The three dimension station coordinate estimates have been converted to position discrepancies in north, east and up components with respect to the true coordinates.

Figures 3 and 4 depict the North, East and Up (NEU) positioning error for $\mathrm{G}$ only and combined GR observations. These figures are obtained using $g L A B$ software. It can be clearly observed from Figure 3 and 4 that the position error for the $\mathrm{G}$ only and mixed GR processing are quite similar in the east and north directions while it is absolutely different in up direction. This is because of vertical error component is always more than one and half times of horizontal error component (Heselton, 1998). After an hour, the positioning errors in both east and north directions converge to a centimeter level while longer time period is required for the vertical component to converge in case of $\mathrm{G}$ only solution. However, for combined
GR solution, a better accuracy will be achieved in a less duration of time.

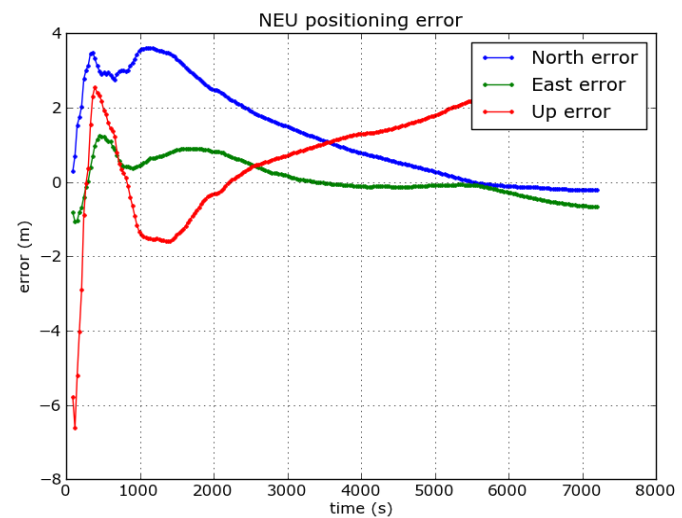

Figure 3: Positioning errors for $\mathrm{G}$ only observation processed by $g L A B$ software

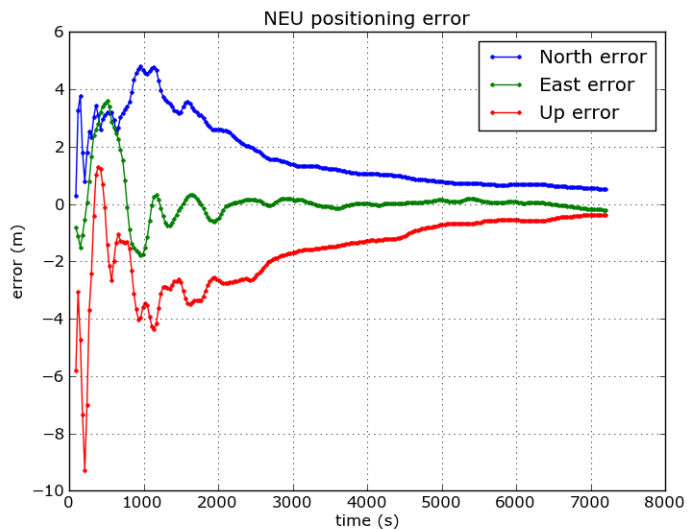

Figure 4: Positioning errors for combined (GR) observation processed by $g L A B$ software

It can also be observed from Figures 3 and 4 that the convergence time of the east, north and up coordinate components in the combined GR processing is smaller than that of the $\mathrm{G}$ only processing because there is an improvement of the convergence time in the vertical coordinate component in case of combined GR solution. Therefore, the overall positioning accuracy of approximately $10 \mathrm{~cm}$ can be achieved with two hrs of observations for the combined GR solution, while it requires more than two hrs for the $\mathrm{G}$ only solution to achieve the same accuracy level. Hence, the overall positioning accuracy enhances due to addition of GLONASS constellation that improves the visibility of satellites as well as satellite geometry.

Figures 5 and 6 depict the satellite geometry as illustrated by the Dilution of Precision (DOP), which can provide valuable information for the analysis, especially when problems occur. These figures are also obtained using $g L A B$ software. The computation of the DOP in the combined GR processing is based on the design matrix with respect to three position components, one receiver clock offset and one system time difference (GPS and GLONASS time), which has one more column when compared with the design matrix for the DOP computation in the $\mathrm{G}$ only processing. The average DOP values of $\mathrm{G}$ only as well as combined GR observations is computed for complete observation period and are shown in table 1. 


\begin{tabular}{|c|c|c|c|c|}
\hline Mode & HDOP & VDOP & GDOP & PDOP \\
\hline G Only & 1.3568 & 1.6239 & 2.3296 & 1.5538 \\
\hline GR & 0.8576 & 1.2296 & 1.6824 & 1.0637 \\
\hline
\end{tabular}

Table 1: Average DOP values

It is calculated that on addition of GLONASS constellation Horizontal Dilution of Precision (HDOP), Vertical Dilution of Precision (VDOP), Geometric Dilution of Precision (GDOP) and PDOP improved by $36 \%, 25 \%, 28 \%$ and $32 \%$ respectively which shows the improvement of satellite geometry by more than $30 \%$.

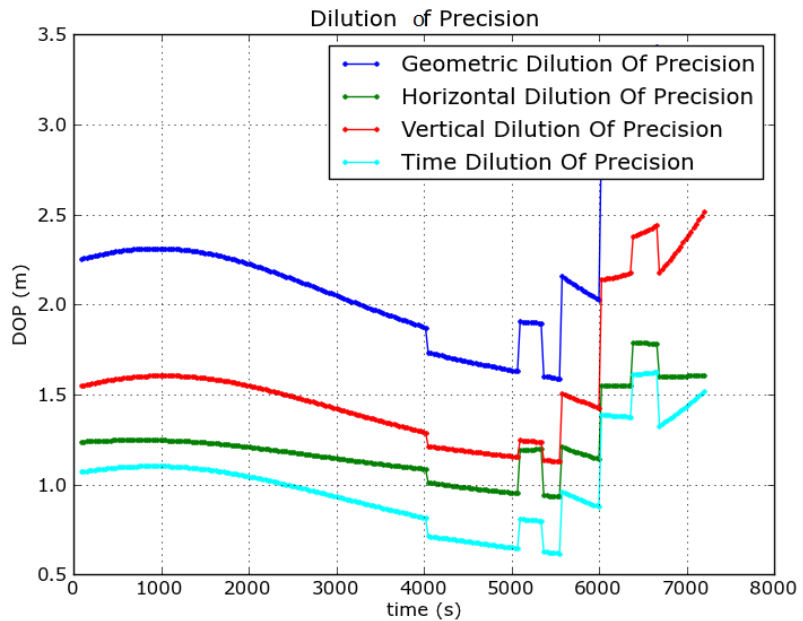

Figure 5: Different DOP values for G only observation processed by $g L A B$ software

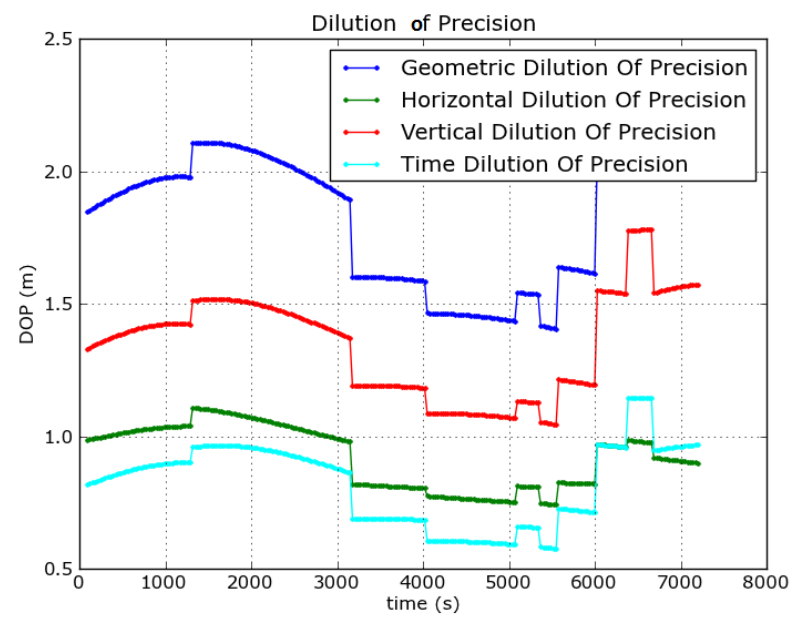

Figure 6: Different DOP values for combined GR observation processed by $g L A B$ software

Figures 7 and 8 illustrate the plot of code and phase residuals with respect to elevation angle for combined GR observations and $\mathrm{G}$ only respectively when elevation angle varies. These results are achieved after processing using magicGNSS software. The code residual plot indicates that the GPS code observations have larger residuals than the combined GR code observations. This is caused by the lower accuracy of the GPS code measurements as well as the satellite orbit and clock corrections. To reduce their effect on the positioning results, the $\mathrm{G}$ only code observations are assigned a smaller weight in the data processing. The phase residual plot also indicates that GPS phase observations have slightly larger residuals than combined GR phase observations, which is obvious particularly for elevation angles between 50 and 60 degrees. RMS values of code and phase observation residuals are also computed by the software. RMS errors for $\mathrm{G}$ only observations are higher than the combined GR observations, which show that the accuracy will increase on adding GLONASS constellation.
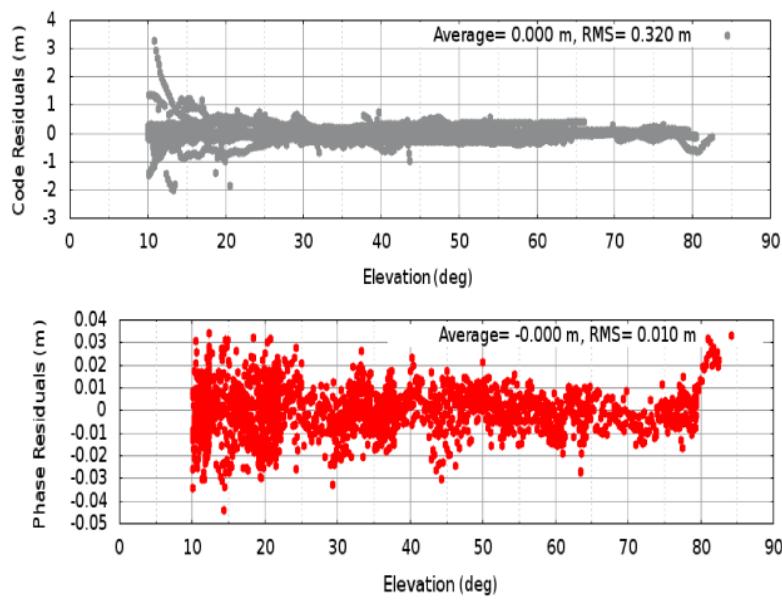

Figure 7: Code and Phase residuals vs elevation angle for combined GR observations
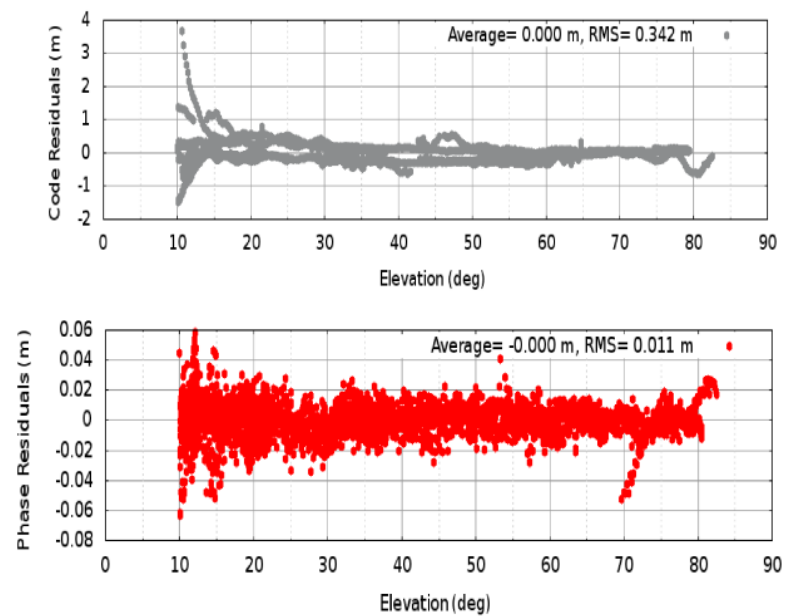

Figure 8: Code and Phase residuals vs elevation angle for $\mathrm{G}$ only observations

Table 2 shows the number of satellites used and rejected in two different modes. During the entire test period, 13 and 21 satellites are used in GPS only and combined GR processing. The observations from approximately 13 GPS satellites and 9 GLONASS satellites on an average are processed for combined GR processing which results that GLONASS constellation improves the visibility of satellites by more than $60 \%$.

\begin{tabular}{|c|c|c|c|}
\hline Mode & Total Satellite & $\begin{array}{c}\text { Used } \\
\text { Satellite }\end{array}$ & $\begin{array}{c}\text { Rejected } \\
\text { Satellite }\end{array}$ \\
\hline G Only & 30 & 13 & 17 \\
\hline GR & 52 & 21 & 31 \\
\hline
\end{tabular}

Table 2: Number of Satellites given by magicGNSS software

Figure 9 and 10 represent the NEU positioning differences between refined and true coordinate for $\mathrm{G}$ only and combined GR observations. These figures are obtained using magicGNSS software. The difference between refined and true coordinates is higher for $\mathrm{G}$ only observations in comparison to combined GR observation. This concludes that the addition of GLONASS 
constellation improves the overall positioning accuracy due to better geometry and visibility of satellites.

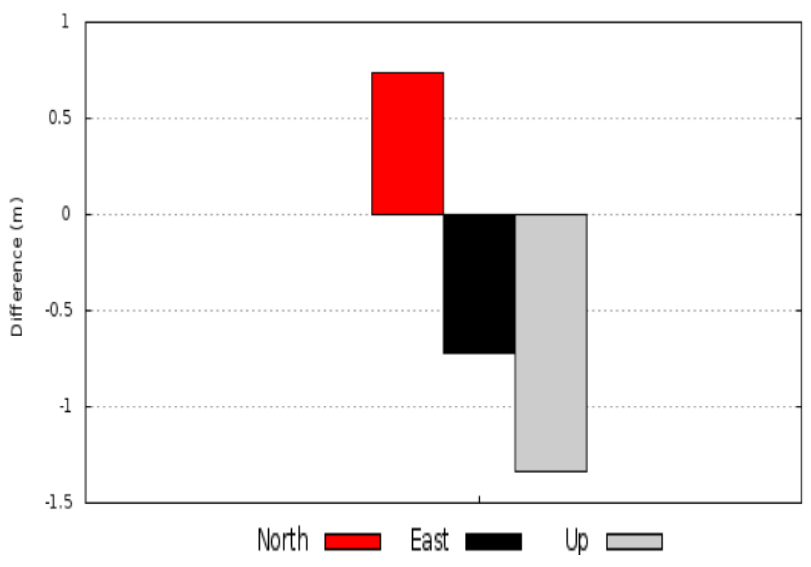

Figure 9: Difference between refined and a priori coordinate for combined (GR) observation processed by magic GNSS software

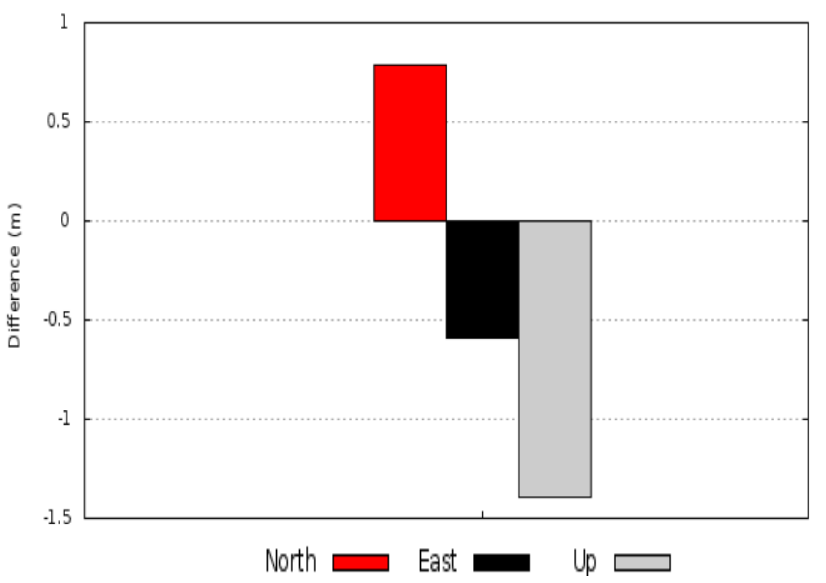

Figure 10: Difference between refined and a priori coordinate for $\mathrm{G}$ only observation processed by magicGNSS software

Finally, it is observed that in comparison to G only solution, the satellite geometry and visibility improves (DOP value decreases) for the case of combined GR while processing the similar data in both open source processing environment i.e. $g L A B$ as well as magicGNSS. Further, on addition of the GLONASS constellation during PPP processing, an enhanced convergence time can be observed which leads to improvement in accuracy. The execution of the mixed GR PPP solution is found to be superior to that of $\mathrm{G}$ only solution.

\section{CONCLUSION}

This study investigated the performance of dual-frequency GPS/GLONASS PPP solution. It has been shown that the addition of GLONASS constellation improves the number of visible satellites by more than $60 \%$ which leads to the improvement of satellite geometry represented by PDOP by more than $30 \%$. This allows for precise surveying in urban areas or when the satellite signal is partially obstructed. In addition, the performance of the combined GR PPP solution was found to be superior to that of $\mathrm{G}$ only solution. Centimeter level accuracy can be achieved within two hours with combined GR PPP solution. However, it requires more than two hours for
G only solution to achieve same accuracy level. Therefore, with more visible GLONASS satellites and the improved GLONASS precise satellite orbit and clock products in the future, the combined GR PPP will be expected to achieve better performance in terms of the overall positioning accuracy with improved solution convergence time. Further improvement can be expected when a full GLONASS constellation is completed in the near future.

\section{RECOMMENDATION FOR FUTURE WORK}

With the availability of Galileo signals in the future, the integration between GPS and Galileo and the integration among GPS, GLONASS and Galileo will be possible for precise point positioning. Also, the performance of the combined GPS and GLONASS PPP needs to be further assessed when more visible GLONASS satellites can be observed and the quality of GLONASS precise satellite orbit and clock products is improved in the future.

\section{REFERENCES}

Abdel-salam, M.A.T., 2005. Precise point positioning using undifferenced code and carrier phase observations, $\mathrm{PhD}$ Thesis, University of Calgary, Canada.

Beutler, G., 2001. Bernese GPS software version 4.2, eds Hugentobler. U., Schaer, S., Fridez, P., Astronomical Institute, University of Berne, Switzerland.

Bisnath, S., 2009. Current state of precise point positioning and future prospects and limitations. In Observing our changing earth (615-623). Springer Berlin Heidelberg.

Cai, C. and Gao, Y., 2007. Precise point positioning using combined GPS and GLONASS observations. Journal of Global Positioning Systems, 6(1): 13-22

Cai, C., 2008. Estimation of GPS-GLONASS system time difference with application to PPP. In Proceedings of ION GNSS, 14(9): 16-19.

Cai, C., 2009. Precise point positioning using dual-frequency GPS and GLONASS measurements. In Masters Abstracts International, 48(03): $172 \mathrm{p}$.

Cai, C. and Gao, Y., 2013. Modeling and assessment of combined GPS/GLONASS precise point positioning. GPS solutions, 17(2): 223-236.

Estey, L.H. and Meertens, C.M., 1999. TEQC: the multipurpose toolkit for GPS/GLONASS data. GPS solutions, 3(1): $42-49$.

Gao, Y., 2009. A combined GPS/GLONASS navigation algorithm for use with limited satellite visibility. Journal of Navigation, 62(04): 671-685.

Gioia, C., 2013. Performance assessment of GPS/GLONASS single point positioning in an urban environment. Acta Geodaetica et Geophysica, 48(2):149-161.

Hernandez-Pajares, M., 2010. The ESA/UPC GNSS-Lab Tool (gLAB). In Proc. of the 5th ESA Workshop on Satellite 
Navigation Technologies (NAVITE 2010), ESTEC, Noordwijk, The Netherlands.

Heroux, P., 2001. Precise point positioning using IGS orbit and clock products. GPS solutions, 5(2): 12-28.

Heselton, R.R., 1998. Elevation effects on GPS positional accuracy, PhD Thesis, Virginia Polytechnic Institute and State University, USA.

Jokinen, A., 2013. GLONASS aided GPS ambiguity fixed precise point positioning. Journal of Navigation, 66(03): 399416.

Langley, R.B., 2010. Analyzing GNSS data in precise point positioning software. GPS solutions, 15(1): 1-13.

Leick, A., 2004. GPS satellite surveying. A Wiley-Interscience publication. USA.

Overby, J., 2000. Global Navigation Satellite System GNSS. Lulea university of technology, Sweden.

Reza G.F., 2006. Online GPS processing services: an initial study. GPS solutions 10(1): 12-20.

Rui, T., 2013. The realization and convergence analysis of combined PPP based on raw observation. Advances in Space Research, 52(1): 211-221.

Sanmartín, M., 2012. Kinematic GNSS-PPP results from various software packages and raw data configurations. Scientific Research and Essays, 7(3): 419-431.

Santerre, R., 2014. Single Point Positioning Using GPS, GLONASS and BeiDou Satellites. Positioning, 5(4):107-114.

Sanz, J., 2011. The ESA/UPC GNSS-Lab Tool (gLAB). An advance educational and professional package for GNSS data processing and analysis, Universitat Politecnica de Catalunya (gAGE/UPC), Barcelona, Spain.

Tsujii, T., 2000. Flight tests of GPS/GLONASS precise positioning versus dual frequency KGPS profile.Earth, planets and space, 52(10): 825-829.

Hofmann-Wellenhof, B., 2007. GNSS-global navigation satellite systems: GPS, GLONASS, Galileo, and more. Springer Science \& Business Media.

Zhang, X., 2014. Integrating GPS and GLONASS to accelerate convergence and initialization times of precise point positioning. GPS solutions, 18(3): 461-471.

Zhu, Y., 2014. SPODS software and its result of precise orbit determination for GNSS satellites. In China Satellite Navigation Conference (CSNC) 2014 Proceedings: Volume III, 301-312). Springer Berlin Heidelberg.

http://gage.upc.edu/gLAB

http://magicgnss.gmv.com/

https://en.wikipedia.org/wiki/Precise_Point_Positioning

http://geodesy.noaa.gov/UFCORS 